\title{
Associations sémantiques et syntaxiques spécifiques. Sur l'exemple du lexique émotionnel des champs de surprise et de déception
}

\author{
Iva Novakova \\ Lidilem (Université Stendhal Grenoble 3) \\ iva.novakova@u-grenoble3.fr \\ Vannina Goossens \\ Lidilem (Université Stendhal Grenoble 3) \\ vannina.goossens@u-grenoble3.fr \\ Elena Melnikova \\ Lidilem (Université Stendhal Grenoble 3) \\ elena.melnikova@u-grenoble3.fr
}

\section{Introduction}

Ce travail a été réalisé dans le cadre du projet ANR franco-allemand Emolex ${ }^{1}$ (en cours) qui porte sur le lexique des émotions dans cinq langues européennes : français, anglais, allemand, espagnol, russe. À partir d'une étude systématique du lexique sur les plans sémantique, syntaxique et discursif, ce projet vise à mieux appréhender la structuration du lexique des émotions dans une perspective interlangue. Notre point de départ est onomasiologique : nous choisissons les champs lexicaux constitués à partir d'une typologie notionnelle (Augustyn et al., 2008). Dans un second temps, l'analyse est sémasiologique : nous étudions les profils combinatoires et fonctionnels des lexies (noms, verbes et adjectifs) faisant partie de ces champs lexicaux.

À la différence des études qui partent de la syntaxe pour étudier le sens (Harris, 1988 ; Gross, 1995 ; le classement des verbes chez Dubois \& Charlier, 1997), ainsi que de celles qui partent d'ensembles sémantiquement homogènes de lexies pour examiner leurs propriétés syntaxiques (Mathieu, 2000 ; Buvet et al., 1995), nous proposons ici une troisième voie. Notre objectif est de montrer qu'il existe des liens spécifiques entre les associations sémantiques des lexies des émotions et les schémas syntaxiques dans lesquels celles-ci se retrouvent. Après une présentation du cadre théorique, de la méthodologie, ainsi que des corpus sur lesquels a été fondée cette étude, nous proposons une analyse sémantico-syntaxique des collocations des champs lexicaux de surprise et de déception, définies dans la tradition anglo-saxonne comme les combinaisons récurrentes entre les mots (Firth, 1957 ; Sinclair, 1991 ; Hoey, 2005). Le terme de collocation est surtout utilisé ici au sens de Hausmann (1979), qui définit cette dernière comme la combinaison phraséologique (codée en langue) d'une base (examen, célibataire, blessé, colère) et d'un collocatif (passer, endurci, grièvement, bouffée de) (Hausmann \& Blumenthal, 2006: 4). Le terme d'émotion, quant à lui, est pris dans un sens large, aussi bien comme renvoyant à des émotions qu'à des sentiments ou à des états affectifs ${ }^{2}$. Dans le cadre de ce travail, il est donc employé plutôt comme une étiquette conventionnelle que comme une sous-catégorisation des affects.

\section{Cadre théorique, méthodologie, corpus}

Nous partons de la théorie du Lexical priming de Hoey (2005). Selon cette théorie, l'emploi d'un mot ou d'une séquence de mots est lié à des associations sémantiques (semantic associations) préactivées (ou amorcées) qui reposent sur le savoir linguistique de chaque locuteur. De plus, Hoey (2005: 44) considère que les items lexicaux sont activés en fonction de leur «préférence » ou «aversion » pour certaines 
fonctions ou environnements grammaticaux (ce qu'il appelle des <colligations>). Nous souhaitons développer et approfondir les propositions de Hoey, appliquées jusqu'alors à un nombre restreint de lexies anglaises. Nous faisons l'hypothèse que les associations entre base (ou mot pivot) et collocatif (par ex. manifester sa surprise, comble d'amertume, très contrarié), qui expriment des dimensions sémantiques comme l'intensité, l'aspect, la causativité ou la manifestation de l'émotion, ont des préférences pour des positions et, de là, pour des fonctions syntaxiques spécifiques (épithète, complément du nom ou du verbe, modifieur du verbe, etc.).

D'un point de vue méthodologique, nous cherchons à combiner deux approches jusqu'à présent exploitées séparément. D'une part, nous proposons une approche sémantico-syntaxique pour l'analyse du lexique des émotions, à travers l'étude de la combinatoire syntaxique et lexicale. L'observation systématique de la combinatoire permet de révéler des propriétés sémantiques et syntaxiques spécifiques des lexies émotionnelles. D'autre part, nous proposons une approche fondée sur des méthodes statistiques (Manning \& Schütze, 1999). Nous considérerons ainsi comme des collocatifs « les accompagnateurs qui s'avèrent préférentiels sur la base du calcul probabiliste log-likelihood» (Blumenthal, 2008: 32). Ce calcul mesure la spécificité des cooccurents des lexies d'un corpus (sur la base de leur lemme), qui est à distinguer de leur fréquence absolue. Cette méthode permet d'établir les relations de proximité distributionnelle et sémantique entre les lexies d'affect dans une langue.

Ce travail est basé sur un vaste corpus de textes contemporains, littéraires et journalistiques, constitué dans le cadre du projet. Ces corpus sont équilibrés dans les cinq langues et contiennent environ 140 millions de mots par langue. De ces corpus sont extraits les lexicogrammes (ou tables de contingence) des lexies analysées, permettant de rendre compte de leur combinatoire syntaxique et lexicale. Le lexicogramme présente les données lexicométriques qui regroupent les paramètres de contingences suivants : fréquence de la cooccurrence du pivot et du collocatif, fréquence de toutes les cooccurrences du pivot, fréquence de toutes les cooccurrences du collocatif, regroupement de tous les cooccurrences présentant une valeur de significativité au moins égale au seuil de spécificité qui a été fixé à 10,83 . Nous pouvons ainsi dresser le profil combinatoire ${ }^{3}$ des lexies d'émotion sélectionnées, c'est-à-dire rendre compte de leur voisinage lexico-syntaxique spécifique. Les cooccurrents sont sélectionnés en deux temps. La première étape de sélection est automatique : nous ne conservons que les collocatifs spécifiques, sur la base du calcul de log-likelihood. La seconde étape est manuelle : nous ne conservons que les cooccurrents des lexies utilisées dans une interprétation d'émotion, et seulement lorsqu'ils forment des collocations avec les mots pivots.

L'analyse est transcatégorielle : les lexies retenues, appartenant aux champs de surprise et de déception, sont des noms, des verbes et des adjectifs, analysées à partir des mêmes grilles sémantique et syntaxique (cf. section 3). Le tableau 1 répertorie l'ensemble des lexies étudiées et indique le nombre total d'associations pivot-collocatif pour chaque champ, après les deux étapes de sélection que nous avons présentées ci-dessus :

\begin{tabular}{|l|l|l|}
\hline Catégorie & Déception & Surprise \\
\hline Noms & Amertume, déception, dépit & Étonnement, surprise, stupeur \\
\hline Verbes & Contrarier, décevoir & Épater, étonner, frapper, surprendre \\
\hline Adjectifs & Contrarié, décevant, déçu & $\begin{array}{l}\text { Étonnant, étonné, frappé, stupéfait, } \\
\text { stupéfiant, surprenant, surpris }\end{array}$ \\
\hline Totaux & 2667 & 7857 \\
\hline
\end{tabular}

Tableau 1 : Nombre d'associations pivot-collocatif

Comme le montre le tableau 1 , les échantillons pour les deux champs ne sont pas équilibrés. Pour cette raison, les calculs statistiques effectués sont fondés sur l'indice de spécificité log-likelihood normalisé, afin de minimiser le biais introduit par ces différences de fréquence. Les valeurs de poids collocationnel sur la base des scores de significativité normalisés sont déterminées par rapport à leurs maxima et minima respectifs et du nombre de collocatifs retenus (Diwersy, 2007: 91) ${ }^{4}$. Les résultats statistiques sont 
présentés sous forme de camemberts qui représentent le poids collocationnel des lexicogrammes enrichis ${ }^{5}$.

Dans la section suivante, nous allons présenter les grilles d'analyse dont nous nous sommes dotés afin de traiter les collocations étudiées.

\section{Grilles d'analyse}

Le codage des lexicogrammes extraits du corpus est effectué à l'aide de deux grilles d'analyses : sémantique et syntaxique.

\subsection{Grille sémantique}

Nous avons élaboré une grille de dimensions sémantiques à partir des travaux de Goossens (2005) et de Tutin et al. (2006). Cette grille propose une liste des dimensions sémantiques que véhiculent les collocatifs des noms, verbes et adjectifs d'émotion. Celle-ci compte huit dimensions sémantiques (manifestation, contrôle, polarité, aspect, intensité, causativité, verbalisation, expérienciation) qui ont chacune des valeurs spécifiques. Le tableau 2 illustre quelques couples de dimensions-valeurs sémantiques.

\begin{tabular}{|l|l|l|}
\hline Dimension & Valeur & Exemple \\
\hline \multirow{2}{*}{ Contrôle } & Émotion & Ravaler sa honte \\
\cline { 2 - 3 } & Manifestation & Dissimuler sa joie \\
\hline \multirow{2}{*}{ Aspect } & Phasique/inchoatif & Commencer à être triste \\
\cline { 2 - 3 } & Phasique/terminatif & Cesser d'avoir peur \\
\hline \multirow{2}{*}{ Polarité } & Interne/positif & Agréablement surpris \\
\cline { 2 - 3 } & Externe $/$ positif & Admiration légitime \\
\hline
\end{tabular}

Tableau 2 : Exemples de dimensions-valeurs sémantiques

Dans ce travail, nous nous intéresserons à quatre dimensions : l'intensité, l'aspect, la causativité et la manifestation.

La dimension intensité permet de regrouper les collocatifs qui apportent des indications quant à l'intensité de l'émotion exprimée, par des moyens parfois métaphoriques. Nous distinguons deux valeurs : fort (mourir de peur, très énervé, une colère immense) et faible (un peu fâché, légèrement surpris, surprendre à peine).

La dimension aspect réunit les collocatifs qui expriment la représentation du déroulement d'une émotion (ou bien la façon dont se déroule une émotion dans le temps). Nous distinguons les collocatifs véhiculant un aspect ponctuel (instant de stupeur) ou duratif (nager dans le bonheur), qui peuvent être ou non itératifs, des collocatifs qui expriment les phases du déroulement de l'émotion : inchoative (commencer à être triste), croissance graduelle (la panique augmente), décroissance graduelle (la tristesse s'adoucit) et terminative (cesser d'avoir peur).

La dimension causativité concerne les collocatifs qui expriment le fait qu'un événement, quelqu'un ou quelque chose amène une personne à ressentir une émotion. Ces collocatifs peuvent être neutres (faire peur, donner de la joie) ou bien exprimer des phases du déroulement de l'émotion et relever également de la dimension 'aspect' : phase initiale (éveiller la crainte), croissance ou décroissance graduelle (attiser la haine, calmer les craintes) ou phase finale (chasser la peur).

La dimension manifestation caractérise les collocatifs qui expriment la façon dont certaines émotions sont extériorisées par l'expérienceur et peuvent être perçues par un tiers. Ces manifestations peuvent être physiques (incluant les expressions du visage), actives (sauter de joie, manifester sa surprise) ou subies (trembler de peur, blêmir d'horreur), ou verbales ${ }^{7}$ (hurler de peur, cri de surprise). Les collocatifs 
peuvent également traduire un jugement extérieur à l'expérienceur : le collocatif exprime le fait qu'une tierce personne a perçu l'émotion ressentie par l'expérienceur par le biais de ses manifestations (deviner la surprise, visiblement surpris).

\subsection{Grille syntaxique}

La grille de relations syntaxiques a été élaborée à partir des travaux de Blumenthal (2007b). Cette grille propose une liste de codes qui permet de rendre compte de la relation syntaxique existant entre un mot pivot et son collocatif. Une même collocation est ainsi codée sémantiquement et syntaxiquement. Les relations syntaxiques sont classées sur la base de la nature syntaxique du mot pivot : la grille contient ainsi trois ensembles de relations syntaxiques, spécifiques aux noms, verbes et adjectifs. Le tableau 3 illustre quelques exemples de relations syntaxiques répertoriées dans notre grille.

\begin{tabular}{|l|l|l|}
\hline Catégorie & Sous-catégorie & Exemple \\
\hline \multirow{2}{*}{$\begin{array}{l}\text { Nom pivot }= \\
\text { complément }\end{array}$} & $\begin{array}{l}\text { N31 : nom (+ préposition) + nom } \\
\text { pivot (sauf quantification) }\end{array}$ & Moment de surprise, larmes de dépit \\
\cline { 2 - 3 } & $\begin{array}{l}\text { N71: nom pivot complément direct/ } \\
\text { prépositionnel du verbe }\end{array}$ & $\begin{array}{l}\text { Atténuer l'amertume, emplir de } \\
\text { stupeur }\end{array}$ \\
\hline \multirow{2}{*}{ Adjectif pivot } & A3: adjectif pivot épithète & Un air surpris \\
\cline { 2 - 3 } $\begin{array}{l}\text { Verbe pivot }+ \\
\text { actants/compléments }\end{array}$ & $\begin{array}{l}\text { V22: verbe pivot + complément } \\
\text { d'objet direct }\end{array}$ & Surprendre tout le monde \\
\cline { 2 - 3 } & $\begin{array}{l}\text { V23: verbe pivot + complément } \\
\text { d'objet indirect }\end{array}$ & S'étonner de son insolence \\
\hline
\end{tabular}

Tableau 3 : Exemples de relations syntaxiques

Dans la section suivante, nous proposons une analyse de la façon dont s'articulent les dimensions sémantiques et les relations syntaxiques des lexies de surprise et de déception afin de tester notre hypothèse selon laquelle les items lexicaux qui véhiculent des dimensions sémantiques spécifiques en fonction de leur combinatoire lexicale ont des préférences pour des positions et, de là, pour des fonctions syntaxiques spécifiques.

\section{Dimensions sémantiques et relations syntaxiques spécifiques des lexies de surprise et de déception}

Nous proposons dans cette section une analyse systématique des relations syntaxiques privilégiées correspondant aux dimensions sémantiques intensité (4.1), aspect (4.2.), causativité (4.3.) et manifestation (4.4.). Nous observerons ces phénomènes pour le français, et montrerons que les collocations relevées en anglais, espagnol et allemand se comportent de façon similaire.

\subsection{Intensité}

Quantitativement, l'intensité est la dimension sémantique la plus importante pour les lexies des champs déception comme surprise. Cette dimension sémantique est transversale : nous trouvons des collocatifs intensifs pour les noms, les adjectifs et les verbes. Néanmoins, l'intensité est majoritairement véhiculée par les pivots adjectivaux $(66 \%$, cf. figure 1). Les collocatifs exprimant l'intensité forte (extrêmement déçu, grosse surprise, étonner grandement) sont nettement plus fréquents $(80 \%)$ que ceux exprimant l'intensité faible (20\%): peu surprenant, légère amertume, décevoir partiellement. Cette dimension sémantique est associée à des constructions syntaxiques privilégiées pour chacune des catégories de mots pivots: modifieurs adverbiaux avec les pivots verbaux ou adjectivaux $(\mathbf{V}+\mathrm{Adv}$ ou $\mathrm{Adv}+\mathbf{A d j})$ et modifieurs adjectivaux avec les pivots nominaux $(\operatorname{Adj}+\mathbf{N})$. 
Pour les adjectifs, la construction syntaxique «modifieur adverbial + adjectif pivot» (Adv+ $\mathbf{A d j})$ est la seule construction impliquée dans l'expression de l'intensité. Dans les exemples (1) et (2), l'adjectif pivot est en fonction d'attribut:

(1) Le maire de la commune, informé hier, s'est dit très contrarié. (Ouest-France)

(2) J'imaginais une usine formide et je suis tout surpris d'atterrir dans des bâtiments importants, nichés au fond d'une ruelle trottoirs. (F. Dard)

La construction « pivot verbal+modifieur adverbial » $(\mathbf{V}+\mathrm{Adv})$ est, comme pour les adjectifs, la seule construction syntaxique utilisée pour marquer l'intensité.

(3) Elle me dit qu'elle se prénomme Natacha, ce qui me surprend beaucoup. (M. Druon)

(4) En attendant, le veto de Bruxelles le contrarie sérieusement. (Le Figaro)

Les noms présentent plus de variétés dans les constructions syntaxiques en général dans nos corpus, et cela se vérifie pour les collocations d'intensité. La construction avec un adjectif épithète antéposé $(\operatorname{Adj}+\mathbf{N})$ est cependant nettement la plus spécifique.

(5) Il y a une énorme attente et une grosse déception à l'origine de la démobilisation de la gauche. (Libération)

(6) Bruno la feuilleta avec une légère surprise, retrouva la page. (M. Houellebecq)

À la marge (secteur «Autres » de la figure 1), nous relevons quelques structures avec un déterminant quantifieur $(\operatorname{Det}+\mathbf{N})$, comme dans l'exemple (7), ou des structures binominales, comme dans l'exemple (8).

(7) J'ai senti beaucoup de déception chez eux, révèle Raphaël Ibanez. (Le Figaro)

(8) «Je croyais qu'ici on parlait d'autre chose» ajoute cette militante de Belgrade avec un brin d'amertume. (Le Monde)

La figure $1^{8}$ montre que l'intensité est, en toute logique, essentiellement exprimée par des modifieurs adverbiaux et adjectivaux.

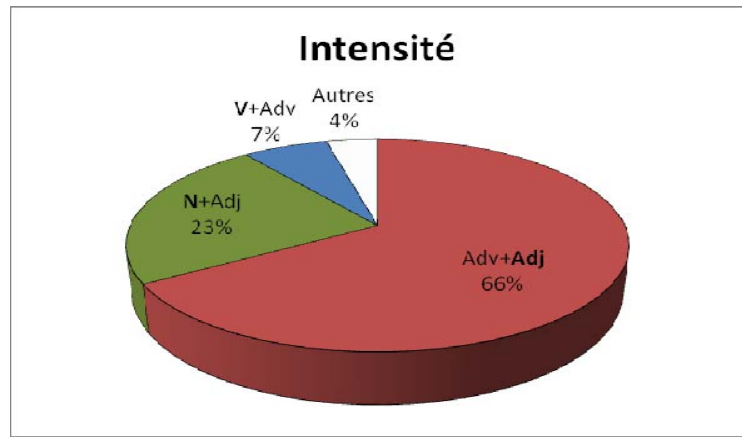

Figure 1 : Les constructions syntaxiques spécifiques de l'intensité ${ }^{9}$

Du point de vue contrastif, nous observons une grande homogénéité dans les structures syntaxiques du français, de l'anglais et de l'espagnol. Dans ces trois langues, l'intensité est majoritairement associée à un pivot adjectival accompagné d'un modifieur adverbial $(\mathrm{Adv}+\mathbf{A d j})$ : más sorprendente 'plus surprenant', (to be) extremely disapointed '(être) extrêmement déçu'. Suivent les pivots nominaux, accompagnés d'un adjectif épithète (Adj+N : crushing disappointment 'écrasante déception', sorpresa mayúscula 'surprise énorme') et les pivots verbaux, accompagnés d'un modifieur adverbial (V+Adv : to greatly astonish 'étonner fortement', decepcionar un tanto 'décevoir quelque peu'). Seul l'allemand présente une configuration de structures syntaxiques légèrement différente. Tout d'abord, ce sont les pivots nominaux 
qui sont les plus spécifiques à l'expression de l'intensité. Pour ceux-ci, la construction la plus fréquente reste Adj+N (eine massive Enttäuschung 'une déception massive') mais nous relevons également des constructions dans lesquelles le nom pivot est le complément d'un adjectif (voller Erstaunen (sein) '(être) plein d'étonnement'), plus fréquentes en allemand que dans les trois autres langues (par ex. en français plein d'amertume). Suivent les pivots adjectivaux et verbaux, dans des proportions égales, qui présentent les mêmes structures que dans les autres langues: Adv+Adj (leicht erstaunt 'légèrement étonné') et $\mathrm{Adv}+\mathbf{V}$ (schrecklich enttäuschen 'décevoir terriblement').

\subsection{Aspect}

Les constructions syntaxiques spécifiques de cette dimension véhiculent les valeurs aspectuelles ponctuelle, durative ou phasique. Ici aussi ce sont les trois catégories (noms, verbes et adjectifs) qui sont concernées mais à des proportions variables (cf. la figure 2).

Pour les verbes pivots, les constructions avec des adverbes modifieurs ( $\mathbf{V}+\mathrm{Adv})$ expriment des valeurs ponctuelles (itératives) et ce, dans les deux champs : surprendre quelquefois, d'emblée, plus d'une fois ; décevoir encore, une nouvelle fois :

(9) Leur légèreté me surprend quelquefois. (P. Cintas)

(10) Marseille a encore déçu, incapable de garder son avance. (Ouest-France)

Cette même construction est également fréquente pour les valeurs duratives : épater, étonner toujours, décevoir toujours.

(11) Nicolas Sarkozy s'étonne toujours de l'image que la société lui renvoie de lui-même : «On me dit que je fais peur ». (Le Figaro)

(12) Or, une promesse non tenue déçoit toujours plus qu'une surprise, même médiocre. (Libération)

Les constructions $\mathrm{V}+\mathbf{V i n f}$, bien que moins fréquentes que les précédentes $(10 \%$ contre $48 \%$ pour les structures $\mathbf{V}+\mathrm{Adv}$, cf. figure 2), véhiculent en général des valeurs duratives dans la phase médiane du procès (surtout dans le champ de surprise) : continuer à surprendre, ne pas finir de surprendre, ne pas cesser d'étonner.

(13) Kadhafi continue de surprendre en Espagne. (Ouest-France)

Comme le montre la figure 2, ce sont les verbes, modifiés par un adverbe aspectuel ou dans des périphrases aspectuelles phasiques, qui sont les plus fréquents dans cette dimension. Les associations verbales spécifiques renvoient à l'aspect ponctuel vs duratif ou aux phases du procès (le plus souvent la phase médiane).

En ce qui concerne les noms pivots, ils apparaissent le plus souvent dans des combinaisons avec des déterminants nominaux ou adverbiaux $(\operatorname{Det}+\mathbf{N})$. Ces constructions expriment l'aspect ponctuel (dans sa valeur itérative) et concernent plutôt le champ de surprise : un lot de surprises, bien des surprises.

(14) Chaque bilan annuel amène son lot de surprises dans le secteur pharmaceutique. (Le Monde)

(15) La brigade fluviale de la capitale trouve bien des surprises au fond de l'eau. (Libération)

Les noms sont également fréquents dans les structures $\mathrm{V}+\mathbf{N}$ (compl. essentiel), qui représentent $8 \%$ de l'ensemble des associations dans cette dimension. Ces combinaisons véhiculent des valeurs terminatives (pour le champ de déception) : effacer toutes les déceptions, digérer ses déceptions.

(16) L'équipe d'Albert Emon devra effacer sa déception si elle veut l'emporter sur l'AS Saint-Etienne. (Le Monde)

Les phases du procès peuvent aussi être exprimées, bien que plus rarement (4\%), par des associations entre le nom pivot et un adjectif $(\mathbf{N}+\mathrm{Adj})$, où l'adjectif épithète renvoie à la phase inchoative en (17) et (18) ou à la phase terminative en (19) : 
(17) Première surprise, les « ebayeurs » n'éprouvent pas nécessairement des plaisirs solitaires. (Ouest-France)

(18) $[\ldots]$ ce qui se prépare, après les belles promesses, c'est une nouvelle déception du pays, une impuissance, une paralysie. (Le Monde)

(19) Jusqu'à cette surprise finale : la candidate socialiste l'emporte non seulement à Rennes, mais d'un cheveu en Ille-et-Vilaine. (Ouest-France)

Contrairement aux valeurs ponctuelles et duratives qui sont essentiellement véhiculées par les verbes pivots modifiés par des adverbes aspectuels (48\% des cas) («colligations positives » dans la terminologie de Hoey, 2005 : 48-49), les valeurs aspectuelles itératives et phasiques (inchoative ou terminative) sont exprimées en majorité par des constructions ayant un pivot nominal

Enfin, pour les adjectifs pivots, des constructions du type adverbe + adjectif pivot (Adv+Adj) ou verbe+adjectif pivot $(\mathrm{V}+\mathbf{A d j})$ apparaissent à proportions égales $(5 \%$ chacune $)$ surtout dans le champ de surprise. Ces constructions expriment essentiellement la valeur ponctuelle :

(20) Une étude plus fine des comportements des internautes livre des résultats parfois surprenants. (Le Monde)

(21) Elle reste étonnée que l'on demande aux chanteurs wagnériens des qualités différentes de celles requises pour Verdi et Puccini. (Le Figaro)

En observant les tendances générales pour l'expression de la dimension aspectuelle, on constate que les valeurs ponctuelles et duratives correspondent essentiellement à des constructions verbales (58\%) contenant des collocatifs aspectuels adverbiaux ou verbaux $(\mathbf{V}+\mathrm{Adv}, \mathrm{V}+\mathbf{V i n f})$. L'aspect ponctuel peut être aussi exprimé, bien que beaucoup plus rarement (10\%), par des pivots adjectivaux comme en (20) et (21). En revanche, les phases aspectuelles ont une préférence pour des constructions nominales du type $\mathbf{N}+$ Adj (première surprise, surprise finale) ou $\mathrm{V}+\mathbf{N}$ (digérer sa déception). Toujours pour les noms pivots, les constructions du type Dét $+\mathbf{N}$ apparaissent avec un poids collocationnel important (13\%) dans l'expression de la valeur ponctuelle (itérative). La figure 2 illustre le poids des différentes constructions syntaxiques qui correspondent à la dimension aspect.

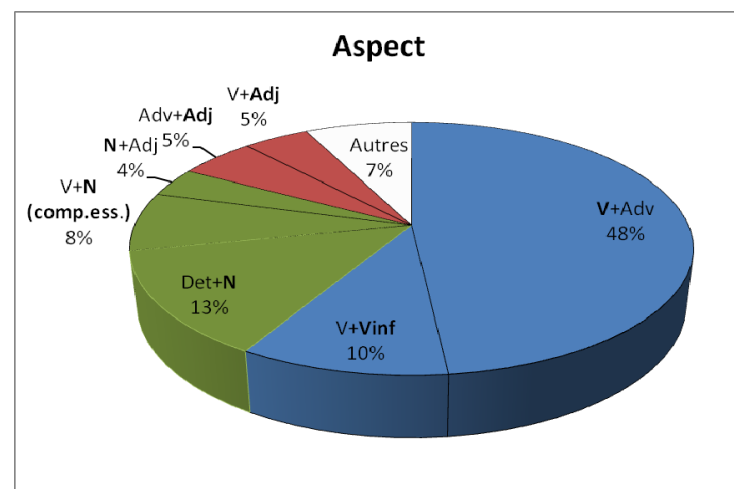

Figure 2 : Les constructions syntaxiques spécifiques de $1^{\prime}$ aspect $^{10}$

Il s'ensuit que l'aspect en français se présente comme une dimension transversale comme l'intensité. A la différence de cette dernière, elle concerne davantage les pivots verbaux et nominaux et moins les pivots adjectivaux. L'aspect regroupe une très grande variété de structures syntaxiques et ce, dans l'ensemble des langues comparées. Les constructions à verbes pivots $(\mathbf{V}+\mathrm{Adv}$ et $\mathrm{V}+\mathbf{V i n f})$ exprimant l'aspect ponctuel ou duratif sont majoritairement présentes en français (58\%) et en anglais (41\%): to surprise again 'surprendre de nouveau', to disappoint rarely 'décevoir rarement', to never cease to amaze 'ne jamais cesser de surprendre'. A la différence de ces deux langues, l'allemand a une préférence pour les 
constructions nominales du type $\mathbf{N}$ (sujet) $+\mathrm{V}$ exprimant l'aspect duratif ou ponctuel (35\%) : der Frust herrscht 'la frustration règne', die Überraschung lauert 'la surprise guette'. On y retrouve aussi, comme en français, la combinaison Det $+\mathbf{N}$ avec un poids collocationnel important (20\%) : viele Überraschungen 'beaucoup de surprises', einige Enttäuschungn 'quelques déceptions'. Les constructions V+N, où le nom pivot est un complément essentiel, caractéristiques de l'aspect phasique, apparaissent aussi en allemand (etw. mündet in Ernüchterung litt.'quelque chose débouche dans le désenchantement') et en espagnol (tornarse en sorpresa 'se transformer en une surprise'). En revanche, les noms modifiés par des adjectifs aspectuels, peu fréquents en français (4\%), présentent des taux plus élevés dans les trois autres langues (entre 11\% et 19\%) : graduel disillusionment 'la désillusion progressive', die einzige Überraschung 'la surprise unique', la decepción continuidado 'la déception continue'. Enfin, l'espagnol fait usage fréquent des constructions adjectivales du type $\mathrm{V}+\mathbf{A d j}(33 \%)$ : quedar, estar decepcionado, estar sorprendido, quedarse perplejo ('rester, être déçu, surpris, perplexe').

Ces tendances illustrent bien le lien entre les valeurs aspectuelles et les structures syntaxiques spécifiques, non seulement en français, mais aussi en espagnol, allemand et anglais. Les valeurs aspectuelles peuvent également se superposer à des valeurs causales.

\subsection{Causativité}

L'analyse des données pour cette dimension montre que ce sont exclusivement les noms pivots (étonnement, surprise, stupeur, amertume et déception) associés à des collocatifs verbaux $(\mathrm{V}+\mathbf{N})$ qui expriment la causativité (neutre). La valeur causative est le plus souvent doublée d'une dimension aspectuelle phasique et, plus rarement, de la dimension intensité. On pourrait classer les collocatifs verbaux en :

- collocatifs causatifs neutres : causer, provoquer la surprise, la déception; réserver une surprise à qqn; susciter l'amertume, la déception;

- collocatifs causatifs aspectuels : inchoatifs (engendrer la déception, plonger dans la stupeur; emplir de stupeur, révéler sa surprise) ou terminatifs (dissiper l'amertume, évacuer la déception) ;

- collocatifs causatifs exprimant à la fois la valeur aspectuelle (croissance graduelle terminative) et l'intensité (faible) : atténuer, adoucir l'amertume ou la déception.

Comme les noms, associés à des collocatifs verbaux causatifs, sont pratiquement les seuls à véhiculer la dimension causative, les constructions syntaxiques spécifiques pour cette dimension sont du type Vcausatif (neutre, aspectuel ou intensif) $+\operatorname{Npivot~}(\mathrm{V}+\mathbf{N})$ en fonction de complément essentiel direct ou indirect (les « colligations positives » de Hoey) : évacuer la déception, emplir de stupeur, plonger dans la stupeur.

(22) Rama Yade, secrétaire d'Etat aux droits de l'homme, n'a pas réussi à adoucir leur amertume. (Le Monde)

(23) Le contrat de vente d'un TGV Tanger-Marrakech atténuera-t-il la déception française ? (Ouest France)

(24) Il en tira une conclusion qui emplit de stupeur ses collaborateurs. (R. Barjavel)

On remarque que les collocations à pivot verbal sont pratiquement absentes dans cette dimension (cf. figure 3) («colligations négatives », Hoey (2005)). Comment expliquer ce fait ? Les verbes surprendre, étonner, décevoir, contrarier appartiennent à la classe II des verbes psychologiques causatifs (Ruwet, 1994 ; Mathieu, 2000). Contrairement aux verbes psychologiques agentifs (classe I) où le sujet est l'expérienceur (celui qui éprouve l'émotion) et le complément d'objet est l'objet du sentiment (Paul aime Marie), les verbes causatifs ont pour sujet la cause de l'émotion, tandis que l'expérienceur en est le complément d'objet (Paul déçoit, surprend, étonne Marie). Ce classement touche de près l'étude systématique des structures actancielles des verbes (leurs actants syntaxiques et les rôles sémantiques 
qu'ils véhiculent), ce qui dépasse l'objet de cette étude ${ }^{11}$. Autrement dit, c'est par leur propre sémantisme (verbe d'émotion causatifs) et par la structure actancielle et non pas par la combinatoire lexicale et syntaxique que les verbes véhiculent la valeur causative. La figure 3 montre que la construction $\mathrm{V}+\mathbf{N}$ est pratiquement exclusive dans l'expression de la causativité en français.

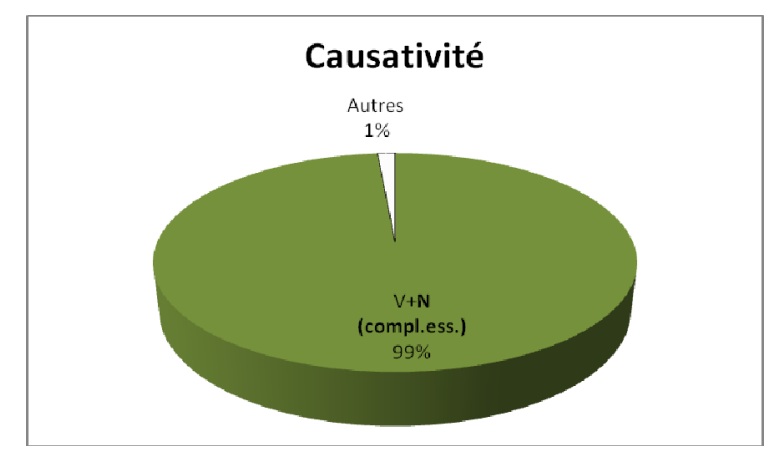

Figure 3 : Les constructions spécifiques de la causativité ${ }^{12}$

La comparaison interlangue montre également que la construction syntaxique $\mathrm{V}+\mathbf{N}$ est majoritaire pour cette dimension en anglais (to cause bewilderment 'causer la perplexité', to compound the disappointment 'aggraver la déception') et en allemand (Verwunderung hervorrufen 'susciter de l'étonnement', für Frust sorgen 'causer la déception'). En espagnol, à côté de $\mathrm{V}+\mathbf{N}$, s'ajoute une autre construction spécifique : il s'agit de l'adjectif pivot (V+Adj) en fonction d'attribut (de l'objet) : dejarle asombrado a alguien 'laisser qqn stupéfait', dejarle perplejo a alguien 'laisser qqn perplexe'. Cette construction (laisser qn stupéfait). existe aussi en français mais reste statistiquement très marginale $(1 \%)$.

(25) Le contenu de ce rapport laisse stupéfaits les professionnels du base-ball. (Libération)

Ces comparaisons montrent que la dimension causative est beaucoup moins transversale que les deux précédentes, car elle concerne presque exclusivement la catégorie nominale, en fonction de complément du verbe.

\subsection{Manifestation}

La dimension manifestation concerne essentiellement les noms et les adjectifs pivots. Pour les noms, elle s'exprime à travers des structures binominales (N1 de N2): traces d'amertume, hurlement de déception, moue, geste, cri, larmes de dépit; murmure, rire d'étonnement; effet, cri, exclamation, rire, hoquet, sursaut de surprise. Le nom pivot est alors complément du N1. Il peut être aussi complément du verbe $(\mathrm{V}+\mathbf{N})$ : afficher, mesurer sa déception; manifester, marquer son étonnement ou sa surprise, exprimer sa stupeur.

(26) Les tenues des Transylvaniens lui arrachèrent un hoquet de surprise [...]. (R.C. Wagner)

(27) [...] l'entraîneur fédéral Grégory Halain pouvait afficher une déception légitime au regard de la prestation de ses protégés. (Le Monde).

Plus rarement, le nom pivot peut apparaître dans les constructions passives où il est complément d'agent (5\%) : être frappé de stupeur ou être pétrifié, figé par la stupeur. Dans ces cas, la valeur manifestation est doublée de la valeur intensité.

(28) A l'instant de ta mort, tu comprendras et seras pétrifié par la stupeur en découvrant de quel néant tu es fait et quel néant tu laisses. (F. Dard)

Pour les adjectifs des deux champs (la classe grammaticale la plus concernée par cette dimension sémantique, à hauteur de $68 \%$ ), ils sont essentiellement en position et, respectivement, en fonction 
d'épithète ( $\mathrm{N}+\mathbf{A d j})$ ou d'attribut ( $\mathrm{V}+\mathbf{A d j})$ : expression contrariée, déçue, étonnée) ; moue déçue ; tête, œil, regard étonné, surpris, stupéfait ; mine, silence stupéfait ; paraître, sembler, se montrer contrarié, déçu, surpris, étonné, stupéfait.

(29) Réponse négative, moue déçue : au Caire, depuis que TPS a renforcé son système antipiratage, rares sont ceux qui captent autre chose que la chaine France 24 et i-Télé. (Libération).

(30) Sous le regard stupéfait de Juliette, je pris sa pince à épiler et je sortis en courant. (M. Pagnol)

(31) Tard dans la soirée, Marie Humbert paraissait déçu. (Libération)

A la marge (secteur «Autres » de la figure 4), les adjectifs pivots peuvent s'associer à des adverbes modifieurs dans la construction $\mathrm{Adv}+\mathbf{A d j}$ (visiblement déçu, étonné, surpris, contrarié), mais le poids collocationnel de ces associations reste assez faible :

(32) Bien que visiblement déçue, Fabienne Keller (41,67\%) n’a plus qu'un mandat de sénateur. (Le Figaro)

(33) Le Hutin, visiblement surpris, regardait de droite à gauche. (M. Druon).

Les manifestations s'expriment donc à travers des structures binominales N1 de N2 (N2 pivot en fonction de complément du N1): exclamation de surprise; des structures $\mathrm{V}+\mathbf{N}$ (complément du verbe) : manifester son étonnement ou des associations entre adjectifs pivots et des collocatifs nominaux $(\mathrm{N}+$ Adj) ou verbaux ( $\mathrm{V}+\mathbf{A d j})$ du type air contrarié; sembler, paraître surpris, contrarié, déçu, ce qui explique les fonctions épithète ou attribut dominantes des adjectifs pivots (cf. figure 4).

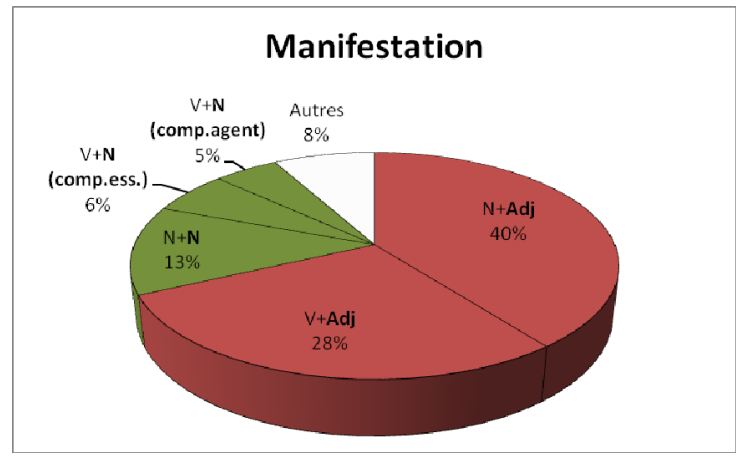

Figure 4 : Les structures syntaxiques spécifiques de la manifestation ${ }^{13}$

L'analyse contrastive montre que la variété des structures syntaxiques observée pour le français se confirme dans les autres langues. Comme en français, le poids collocationnel des structures syntaxiques à pivot adjectival (en fonction d'attribut et d'épithète) est très important en anglais (to look amazed 'paraître stupéfait', to seem desappointed 'paraître déçu', surprised puff 'un souffle surpris') et en allemand: (sich verwundert zeigen 'se montrer étonné', erstaunte Augen 'les yeux étonnés'). En espagnol ce sont les adjectifs attributs qui dominent (45\%) (parecer perplejo 'paraître perplexe'). En anglais, à la différence des trois autres langues comparées, on observe aussi de nombreux adjectifs (20\%) modifiés par un adverbe exprimant la manifestation $(\mathrm{Adv}+\mathbf{A d j})$ : clearly disappointed 'clairement déçu'. Pour les noms pivots, les structures binominales $(\mathrm{N}+\mathbf{N})$ sont les plus fréquentes (moue de dépit, cry of astonishment 'cri d'étonnement', Ausruf des Erstaunens 'exclamation d'étonnement') sauf en espagnol où la fréquence de construction $\mathrm{V}+\mathbf{N}$ complément essentiel est la plus élevée (40\%) (reflejar el desconcierto 'refléter la confusion'). La tendance générale qui s'en dégage malgré ces variations de structures, c'est la prédominance des associations entre adjectifs et noms pivots avec des collocatifs exprimant la manifestation et ce, dans les quatre langues, tandis que les verbes pivots semblent très peu concernés par cette dimension (colligations négatives). 
Pour résumer, l'analyse interlangue pour les quatre dimensions sémantiques étudiées dans ce travail montre qu'il existe une grande similitude entre les associations lexicales réparties dans des dimensions sémantiques et leurs distributions syntaxiques. Pour l'intensité, il s'agit essentiellement de collocatifs adjectivaux et adverbiaux en fonctions d'épithètes ou de modifieurs (très contrarié, contrarier sérieusement, légère surprise). Les collocatifs qui véhiculent des informations aspectuelles sont essentiellement des adverbes modifieurs (surprendre quelquefois) et des verbes aspectuels, suivis d'un nom d'émotion pivot en fonction de complément essentiel du verbe (effacer sa déception). Les adjectifs pivots bien que moins concernés par cette dimension, peuvent être modifiés par des adverbes aspectuels (des résultats parfois surprenants). La dimension causative est exprimée de façon presque exclusive par des collocatifs verbaux causatifs, associés aux noms pivots d'émotion (compléments essentiels) comme susciter l'étonnement, adoucir l'amertume. Enfin, pour la manifestation, comme pour l'aspect, une plus grande variété de structures est observée pour les mots pivots : structures binominales (larmes de dépit), constructions verbo-nominales ou attributives (manifester son étonnement; se montrer déçu), ainsi que des associations entre noms et adjectifs pivots (épithètes) (expression contrariée). Ces résultats confirment notre hypothèse sur le lien étroit entre associations sémantiques et constructions syntaxiques spécifiques.

Par ailleurs, une étude comparative ponctuelle sur les verbes synonymes étonner et surprendre que nous avons menée en français montre que ces verbes, bien que proches sémantiquement, présentent des configurations de dimensions sémantiques très différentes. Ainsi, les collocatifs les plus spécifiques au verbe surprendre sont nettement ceux véhiculant l'expression de la polarité (surprendre agréablement), avant l'aspect (continuer de surprendre) et l'intensité (surprendre beaucoup). À l'inverse, le verbe étonner est d'abord accompagné de collocatifs aspectuels (ne pas finir d'étonner), puis de collocatifs exprimant le contrôle (feindre de s'étonner), l'intensité (étonner fort) et les manifestations (sembler s'étonner). La polarité est totalement absente. Malgré des profils sémantiques très différents, les constructions syntaxiques à l'intérieur des dimensions sémantiques sont identiques pour ces deux verbes : l'intensité et la polarité entrent exclusivement dans des structures $\mathbf{V}+\mathrm{Adv}$ (surprendre beaucoup, davantage, agréablement, positivement, étonner fort, vraiment), l'aspect accepte les structures $\mathbf{V}+\mathrm{Adv}$ et $\mathrm{V}+$ Vinf (surprendre souvent, étonner toujours, ne pas finir de surprendre, continuer d'étonner), etc. Nous pouvons faire des observations identiques dans le champ déception, pour les verbes contrarier et décevoir. Les collocatifs spécifiques du verbe contrarier $^{14}$ véhiculent l'intensité (contrarier fortement) puis, dans une moindre mesure, la manifestation (contrarier manifestement). Les collocatifs de décevoir véhiculent des dimensions sémantiques plus variées : l'intensité (décevoir profondément), la polarité (décevoir amèrement), l'aspect (décevoir souvent) et, de manière plus marginale, la manifestation (décevoir visiblement). Cependant, les structures syntaxiques sont une fois de plus très homogènes pour les associations pivot-collocatif de ces deux verbes : l'intensité comme les manifestations (qui sont les deux dimensions sémantiques communes à ces verbes) sont véhiculées exclusivement par des structures $\mathbf{V}+\operatorname{Adv}$ (décevoir horriblement, contrarier davantage, décevoir visiblement, contrarier manifestement), comme nous l'avons montré pour les verbes du champ surprise. Ce fait conforte sous un autre angle l'hypothèse du lien entre associations sémantiques et syntaxiques spécifiques. Cette étude que nous envisageons d'approfondir présente des pistes intéressantes pour la suite de ce travail.

\section{Conclusion}

L'étude qualitative que nous avons menée sur les lexies des champs de surprise et de déception, confortée par des données statistiques, apporte une preuve convaincante en faveur de la théorie du Lexical priming de Hoey (2005) : les collocations ont des préférences marquées ou inversement des «aversions » pour des constructions syntaxiques particulières. Nous avons cependant voulu aller plus loin. A la différence de Hoey, nos associations lexicales sont d'abord classées dans des dimensions sémantiques au sein desquelles sont identifiées des relations syntaxiques. Cette démarche montre que l'étude de la combinatoire lexicale est indissociable de celle de la combinatoire syntaxique. Deux principaux résultats se dégagent sur le plan théorique. D’une part, nous avons établi que les associations lexicales spécifiques qui sont très variées au sein de chaque dimension sémantique et ce, pour les lexies des deux champs, 
correspondent à des constructions syntaxiques régulières (fonctions grammaticales préférées ou évitées), dont le nombre est plus limité. C'est une tendance générale, confirmée par les études quantitatives intralangues, ainsi que par nos comparaisons qualitatives interlangues. D'autre part, des lexies synonymes au sein d'un même champ lexical (cf. supra pour étonner et surprendre ou contrarier et décevoir) dans une langue peuvent présenter des profils très différents du point de vue sémantique mais ne se distinguent pas réellement du point de vue des constructions syntaxiques propres à ces dimensions sémantiques. Autrement dit, une lexie pourra être accompagnée de différents collocatifs intensifs par exemple, mais pour toutes les lexies ces collocatifs auront les mêmes relations syntaxiques privilégiées.

Plus généralement, ce travail confirme le constat de Hoey, à savoir que la grammaire est un filtre qui organise et «discipline» le lexique (Hoey, 2005 : 159-162). Cette étude lexico-grammaticale du lexique émotionnel plaide en faveur de la non séparation de la syntaxe et du lexique (c'est aussi le cas des grammaires de construction Fillmore et al., 1988; Goldberg, 1995) et d'une «dissolution » de la distinction entre études lexicales et grammaticales du lexique (cf. Hoey, 2005).

\section{Références bibliographiques}

Augustyn, M., Ben Hamou, S., Bloquet, G., Goossens, V., Loiseau, M. \& Rynck, F. (2008). Constitution de ressources pédagogiques numériques : le lexique des affects. In Loiseau, M., Abouzaïd, M., Buson, L., Cavalla, C., Djaroun, A., Dugua, C., Ghimenton, A., Goossens, V., Lebarbé, T., Nardy, A., Rinck, F. \& Surcouf, C. (eds.). Autour des langues et du langage : perspective pluridisciplinaire. Grenoble : Presses Universitaires de Grenoble, 407-414.

Blumenthal, P. (2007a). Sciences de l'Homme vs sciences exactes: combinatoires des mots dans la vulgarisation scientifique, Revue française de linguistique appliquée, 12-2, 15-28.

Blumenthal, P. (2007b). A Usage-based French Dictionary of Collocations. In Kawaguchi, Y., Takagaki, T., Tomimori, N. \& Tsuruga, T. (eds.). Corpus-Based Perspectives in Linguistics. Amsterdam : Benjamins, 67-83.

Blumenthal, P. (2008). Histoires de mots : affinités (s)électives. In Durand, J., Habert, B. \& Laks, B. (eds.). Congrès Mondial de Linguistique Française 2008. Institut de Linguistique Française, [en ligne] : http://dx.doi.org/10.1051/cmlf08338, 31-46.

Buvet, A., Girardin, C., Gross, G. \& Groud, C. (2005). Les prédicats d'<affect>. Lidil, 32, 123-143.

Diwersy, S. (2007). Kookkurrenz, Kontrast, Profil : Korpusinduzierte Studien zur lexikalischsyntaktischen Kombinatorik französischer Substantive (mit ergänzenden Betrachtungen zum Deutschen). Diss. Romanisches Seminar, Universität zu Köln.

Dubois, J. \& Dubois-Charlier, F. (1997). Les Verbes français. Paris : Larousse-Bordas.

Fillmore, C., Kay, P., O'Connor, C. (1988). Regularity and idiomaticity in grammatical constructions: the case of let alone. Language, 64, 501-538.

Firth, J.R. (1957). Papers in Linguistics. London: Oxford : University Press.

Flaux, N. \& Van de Velde, D. (2000). Les noms en français: esquisse de classement. Gap/Paris : Ophrys.

Goldberg, A. E. (1995). Constructions. A Construction Grammar Approach to Argument Structure. Chicago: University of Chicago Press.

Goossens, V. (2005). Les noms de sentiment : esquisse de typologie sémantique fondée sur les collocations verbales. Lidil, 32, 103-121.

Gross, M. (1995). Une grammaire locale de l'expression des sentiments. Langue Française, 105, 70-87.

Harris, Z.S. (1988). Language and Information. New York : Columbia University Press.

Hausmann, F. (1979). Un dictionnaire des collocations est-il possible ? Travaux de littérature et de linguistique de l'université de Strasbourg, XVII/1, 187-195.

Hausmann, F. \& Blumenthal, P. (2006). Présentation : collocation, corpus, dictionnaires. Langue française, 150, 313. 
Hoey, M. (2005). Lexical priming: a new theory of words and language. London/New York : Routledge.

Manning, C. \& Schütze, H. (1999). Foundations of Statistical Natural Language Processing. Cambridge : MIT Press.

Mathieu, Y.Y. (2000). Les verbes de sentiment : de l'analyse au traitement automatique. Paris : Éditions du CNRS.

Novakova, I., Goossens, V. \& Grossmann, F. (à paraître). Interactions entre profil discursif et structures actancielles : l'exemple des verbes de surprise et de respect. Langue Française.

Novakova, I., Grossmann, F. \& Goossens, V. (à paraître). Les structures actancielles des noms de surprise et de respect : analyse fonctionnelle. In Actes du Colloque international Emotion, Cognition and Communication, Nicosie, 23-26 juin 2011.

Ruwet, N. (1994). Etre ou ne pas être un verbe de sentiment. Langue française, 103, 45-55.

Sinclair, J.-M. (1991). Corpus, Concordance, Collocation. Oxford : Oxford University Press.

Tutin, A., Novakova, I., Grossmann F. \& Cavalla, C. (2006). Esquisse de typologie des noms d'affect à partir de leurs propriétés combinatoires. Langue française, 150, 45-55.

1 Le projet franco-allemand ANR/DFG Emolex (ANR-09-FASHS-017), sous la responsabilité d'I. Novakova (Université Stendhal, Grenoble 3) et de P. Blumenthal (Université de Cologne), a pour objectif l'étude du lexique des émotions dans cinq langues européennes dans ses aspects sémantique, syntaxique et discursif.. Site : www.emolex.eu

${ }^{2}$ Buvet et al. (1995) distinguent trois hyperclasses d'affect : <émotion> (colère, enthousiasme), <sentiment> (amour, jalousie), <humeur > (morosité). Pour la distinction entre émotions, sentiments ou états affectifs, cf. entre autres Flaux \& Van de Velde (2000), Goossens (2005), Tutin et al. (2006).

3 Blumenthal (2007a) définit le profil combinatoire d'un mot comme «l'ensemble de ses accompagnateurs stéréotypés, porteurs d'associations typiques ».

${ }^{4}$ Pour le calcul du log-likelihood normalisé, un outil a été créé par S. Diwersy (université de Cologne) dans le cadre du projet Emolex qui fonctionne sous le logiciel $\mathrm{R}$ et qui est implémenté dans une base de données appelée Emogrammes http://santenay.rom.uni-koeln.de/html/emogrammes-v1/

${ }^{5}$ Les lexicogrammes enrichis sont obtenus à partir des lexicogrammes bruts, extraits à partir des corpus multilingues du projet Emolex, après codage selon nos grilles syntaxiques et sémantiques.

${ }^{6}$ La valeur externe est attribuée aux collocatifs qui expriment un jugement de l'émotion par rapport à une adéquation morale ou sociale de celui-ci à la situation.

${ }^{7}$ Nous distinguons les manifestations verbales (hurler de peur) des verbalisations (hurler sa peur) qui constituent une dimension sémantique en elles-mêmes, pouvant véhiculer différentes valeurs (émotives ou communicatives). Contrairement aux collocatifs de manifestation verbale, les collocatifs de verbalisation véhiculent un contenu sémantique.

${ }^{8}$ Les trois couleurs des graphiques renvoient aux catégories des mots pivots : bleu pour les verbes, vert pour les noms, rouge pour les adjectifs.

${ }^{9}$ Le secteur « Autres » de la dimension intensité réunit les constructions N+N, $\operatorname{Adj}^{+}{ }_{\text {prep }}+\mathbf{N}, \operatorname{Det}+\mathbf{N}, \mathbf{N}_{\text {sujet }}+\mathrm{V}$.

${ }^{10}$ Le secteur « Autres » de la dimension aspect réunit les constructions $\mathrm{V}+\mathbf{N}_{\text {comp.agent }}, \mathrm{V}+\mathbf{N}_{\text {comp.n-ess. }}, \mathbf{N}_{\text {sujet }}+\mathrm{V}, \mathrm{N}+\mathbf{N}$.

${ }^{11}$ Pour une étude détaillée des structures actancielles des verbes et des noms des champs de surprise et de respect, cf. Novakova, Goossens, Grossmann (à paraître) et Novakova, Grossmann, Goossens (à paraître).

${ }^{12}$ Le secteur « Autres » de la dimension causativité réunit les constructions $\mathrm{V}+\mathbf{A d j}_{\text {attr }}$.

${ }^{13}$ Le secteur « Autres » de la dimension manifestation rassemble les constructions V+Adv, V+Vinf, Adv+Adj, Adj+ prep $+\mathbf{N}$.

${ }^{14}$ Contrarier possède cependant trois fois moins de collocatifs spécifiques que décevoir. 